\title{
Probing non-standard gravity with the growth index of cosmological perturbations
}

\author{
Heinrich Steigerwald* \\ Aix-Marseille University \\ E-mail: heinrich.steigerwaldecpt.univ-mrs.fr
}

This talk is based on [1]. The growth index of cosmological perturbations is one of the most performant probes of the nature of Dark Energy (DE), the mysterious mechanism driving the late epoch acceleration of the universe. At variance with classical geometrical observables, such as distances, which only probe the background sector of a cosmological model, this observable provides insight into first order dynamical effects, and it is therefore a key test for modified gravity scenarios often invoked to explain away the DE issue.

In this talk I will first show how the whole information about the growth rate history of linear cosmic structures can be precisely encoded into a small set of growth index parameters whose amplitude can be analytically predicted by theory. Then I will go on demonstrating how these parameters naturally define a space where theoretical predictions can be compared against data in a model independent way. Finally [2], by exploiting the Effective Field Theory of dark energy, a formalism which describes virtually all the gravitational theories containing a single scalar degree of freedom in addition to the metric, I will explore which alternative cosmological scenarios are not in conflict with current growth data.

References

[1] H. Steigerwald, J. Bel, C. Marinoni, Probing non-standard gravity with the growth index: a background independent analysis, (accepted by JCAP) [arXiv:1403.0898].

[2] F. Piazza, H. Steigerwald, C. Marinoni, Phenomenology of dark energy: exploring the space of theories with future redshift surveys, (accepted by JCAP) [arXiv:1312.6111]

Frontiers of Fundamental Physics 14 - FFP14,

15-18 July 2014

Aix Marseille University (AMU) Saint-Charles Campus, Marseille

${ }^{*}$ Speaker. 